\title{
Numerical Approximation using Evolution PDE Variational Splines
}

\author{
Abdelouahed Kouibia (D), ${ }^{1}$ Miguel Pasadas, ${ }^{1}$ Zakaria Belhaj $^{2}$ \\ ${ }^{1}$ Department of Applied Mathematics, University of Granada, 18071 Granada, Spain \\ ${ }^{2}$ Laboratory of Study and Research for Applied Mathematics (LERMA), Engineering \\ Mohammedia School, Rabat Morocco
}

Received 18 May 2016; accepted 26 April 2017

Published online 29 May 2017 in Wiley Online Library (wileyonlinelibrary.com).

DOI 10.1002/num.22168

\begin{abstract}
This article deals with a numerical approximation method using an evolutionary partial differential equation (PDE) by discrete variational splines in a finite element space. To formulate the problem, we need an evolutionary PDE equation with respect to the time and the position, certain boundary conditions and a set of approximating points. We show the existence and uniqueness of the solution and we study a computational method to compute such a solution. Moreover, we established a convergence result with respect to the time and the position. We provided several numerical and graphic examples of approximation in order to show the validity and effectiveness of the presented method. (c) 2017 Wiley Periodicals, Inc. Numer Methods Partial Differential Eq 34: 5-18, 2018
\end{abstract}

Keywords: approximation; finite elements; interpolation; PDE; splines; surfaces

\section{INTRODUCTION}

In Computer-Aided Design (CAD), the construction of curves and/or surfaces is done traditionally using geometric primitives such as lines, cones and other types of shapes which can be characterized by some simple equations.

However, when faced with complex problems in engineering, architecture, and geology, what is needed are smooth curves and/or surfaces, which are shapes that cannot be described by a simple equation. Commonly, these problems appear in the automotive or aerospace industries where the section of manufactured objects are designed from some interpolation or approximation data, and also verifying some hydrodynamic properties that can be modeled by certain ordinary differential equation (ODE) and/or partial differential equation (PDE). Hence, it is natural to use free-form curves. In [1], the authors present a design method for free-form curves from a set of approximation points and a boundary value problem for an ODE. In [2], the authors present a general scheme for using PDEs to solve surface modeling problems with high-order boundary continuity conditions.

Correspondence to: A. Kouibia, Department of Applied Mathematics, University of Granada, 18071 Granada, Spain (e-mail: kouibia@ugr.es) 
This article deals with a numerical solution of evolutionary partial differential equation problem respect to the time and the position, by discrete variational splines in a finite element space. A discretization problem in a suitable finite element space is studied. We show the existence and the uniqueness of the solution of such problem and we study a computational method to compute such solution. Then, we establish a convergence result with respect to the time and position. Finally, we analyze some numerical and graphical examples to show the validity and effectiveness of our method. We want to emphasize that this manuscript is entirely different of [3], because we solve the problem in a finite element space at the same time with respect to the time and the position, that is, solving a three-dimensional problem; while in [3] first, we resolve the problem with respect to the time with the finite differential method, second, we resolve the problem in a finite dimensional space with respect to the position. Working in a space of 3D finite element is more difficult, which gives more value to the manuscript due to multiple applications in various fields of science and design.

The remainder is organized as follows. In Section 2, we introduce some notations used in the article; Section 3 is devoted to formulate the evolutionary problem; then in Section 4, we study the resolution of such problem in a finite element space, in Section 5, we give a detailed description of the computation of the method; and we study the convergence of a discrete solution in Section 6. The Section 7 is finished by presenting some numeric and graphical examples to show the validity of the method.

\section{PRELIMINARIES}

The following notations are used:

- Let $n, m \in \mathbb{N}$.

- The Euclidean norm and inner product in $\mathbb{R}^{m}$ will be denoted by $\langle\cdot\rangle_{m}$ and $\langle\cdot, \cdot\rangle_{m}$, respectively, for $m \geq 2$.

- $\Omega$ is an open bounded subset of $\mathbb{R}^{2}$ and $L^{2}(\Omega)$ stands for the linear space of real Lebesgue measurable functions such that $\int_{\Omega} u^{2}(\boldsymbol{x}) d \boldsymbol{x}<+\infty$.

- $H^{n}(\Omega)$ represents the usual Sobolev space $H^{n}(\Omega)$ of order $n$ of (classes of) functions $u \in L^{2}(\Omega)$, together with all their partial derivatives $\partial^{i} u$, in the distribution sense, of order $|\boldsymbol{i}| \leq n$, where for all $\boldsymbol{i}=\left(i_{1}, i_{2}\right) \in \mathbb{N}^{2},|\boldsymbol{i}|=i_{1}+i_{2}$ and $\partial^{i} u(\boldsymbol{x})=\frac{\left.\partial^{|i|}\right|_{u}}{\partial x_{1}{ }_{1} \partial x_{2}{ }^{i_{2}}}$, for any $\boldsymbol{x}=\left(x_{1}, x_{2}\right) \in \Omega ;$

- $H_{0}^{n}(\Omega)$ is the closure of $C_{0}^{\infty}(\Omega)$ in $H^{n}(\Omega)$. Obviously $H_{0}^{0}(\Omega)=L^{2}(\Omega)$;

- Finally, with the same letter $C$ we denote various strictly real positive constants.

The linear space $L^{2}(\Omega)$ is equipped with the inner product

$$
(u, v)_{0}=\int_{\Omega} u(\boldsymbol{x}) v(\boldsymbol{x}) d \boldsymbol{x}
$$

and the corresponding norm $|u|_{0}=(u, u)_{0}^{\frac{1}{2}}$.

Analogously, the Sobolev space $H^{n}(\Omega)$ is equipped with the inner product

$$
((u, v))_{n}=\sum_{|i| \leq n} \int_{\Omega} \partial^{i} u(\boldsymbol{x}) \partial^{i} v(\boldsymbol{x}) d \boldsymbol{x},
$$


the corresponding norm $\|u\|_{n}=((u, u))_{n}^{\frac{1}{2}}$, the semi-inner products

$$
(u, v)_{l}=\sum_{|i|=l} \int_{\Omega} \partial^{i} u(\boldsymbol{x}) \partial^{i} v(\boldsymbol{x}) d \boldsymbol{x}, 0 \leq l \leq n,
$$

and the corresponding seminorms $|u|_{l}=(u, u)_{l}^{\frac{1}{2}}, 0 \leq l \leq n$.

For greater clarity, we will denote $\|\cdot\|_{n, \Omega},((\cdot, \cdot))_{n, \Omega},|\cdot|_{l, \Omega},(\cdot, \cdot)_{l, \Omega}$ instead of $\|\cdot\|_{n},((\cdot, \cdot))_{n},|\cdot|_{l}$, and $(\cdot, \cdot)_{l}$, respectively.

Later, $\mathbb{R}^{m, l}$ is used to signify the space of real matrices with $m$ rows and $l$ columns, endowed with the inner product $\langle A, B\rangle_{m, l}=\sum_{i, j=1}^{m, l} a_{i j} b_{i j}$, where $A=\left(a_{i j}\right)_{\substack{1 \leq i \leq m \\ 1 \leq j \leq l}}$ and $B=\left(b_{i j}\right)_{\substack{1 \leq i \leq m \\ 1 \leq j \leq l}}$, and the corresponding norm $\langle A\rangle_{m, l}=\langle A, A\rangle_{m, l}^{\frac{1}{2}}$.

\section{FORMULATION OF THE PROBLEM}

Now, let $\Omega$ be an open bounded polygonal subset of $\mathbb{R}^{2}$ and $T$ a non-negative real number. Thus, $\Omega$ has a Lipschitz boundary. For simplicity of notation, we write $(0, T) \times \Omega=\Omega_{T}$.

Let $L_{x}$ be a differential operator defined from $H^{2}(0, T) \times H^{2 n}(\Omega)$ into $L^{2}\left(\Omega_{T}\right)$ by

$$
L_{x} u(t, \boldsymbol{x})=\sum_{|i|,|j| \leq n}(-1)^{|j|} \partial_{x}^{j}\left(p_{i j}(\boldsymbol{x}) \partial_{x}^{i} u(t, \boldsymbol{x})\right),(t, \boldsymbol{x}) \in \Omega_{T},
$$

where $p_{i j} \in C^{|j|}(\Omega)$ and $p_{i j}=p_{j i}$ for all $|\boldsymbol{i}|,|\boldsymbol{j}| \leq n$.

We consider the symmetric bilinear form associated with $L_{x}$, defined on $H^{1}(0, T) \times H^{n}(\Omega)$ by

$$
(u, v)_{L_{x}}=\sum_{|i|,|j| \leq n}\left(p_{i j}(\boldsymbol{x}) \partial_{x}^{i} u(t, \boldsymbol{x}), \partial_{x}^{j} v(t, \boldsymbol{x})\right)_{0, \Omega_{T}}
$$

this means that

$$
(u, v)_{L_{x}}=\int_{0}^{T} \int_{\Omega}\left(\sum_{|i|,|j| \leq n}\left(p_{i j}(\boldsymbol{x}) \partial_{x}^{i} u(t, x) \partial_{x}^{j} v(t, x)\right) d \boldsymbol{x} d t .\right.
$$

We assume that

$$
\sum_{|i|,|j| \leq n-1} \xi^{i} p_{i j}(x) \xi^{j} \geq 0, \quad \forall x \in \Omega
$$

and that there exists $v>0$ such that

$$
\sum_{|i|,|j|=n} \xi^{i} p_{i j}(x) \xi^{j} \geq v\langle\xi\rangle_{2}^{2 n}, \quad \forall x \in \Omega
$$

for all $\boldsymbol{\xi}=\left(\xi_{1}, \xi_{2}\right) \in \mathbb{R}^{2}$, where $\xi^{i}=\xi_{1}^{i_{1}} \xi_{2}^{i_{2}}$, for any $\boldsymbol{i}=\left(i_{1}, i_{2}\right) \in \mathbb{N}^{2}$.

Due to (3) and (4), the differential operator $L_{x}$ is said to be strongly elliptic on $\Omega$.

It can be easily shown that according to the hypotheses (3) and (4) the bilinear form $(\cdot, \cdot)_{L_{x}}$ defines a semi-inner product on $H^{n}(\Omega)$. 
The seminorm associated to $(\cdot, \cdot)_{L_{x}}$ is denoted by $|u|_{L_{x}}=(u, u)_{L_{x}}^{\frac{1}{2}}$.

Likewise, let $L_{t}$ be a differential operator defined from $H^{2}(0, T) \times H^{2 n}(\Omega)$ into $L^{2}\left(\Omega_{T}\right)$ by

$$
L_{t} u(t, \boldsymbol{x})=\frac{\partial}{\partial t}\left(p(t, \boldsymbol{x}) \frac{\partial}{\partial t} u(t, \boldsymbol{x})\right),(t, \boldsymbol{x}) \in \Omega_{T},
$$

where $p(t, \boldsymbol{x}) \in C^{1}(0, T), p(t, \boldsymbol{x})>0$, for any $(t, \boldsymbol{x}) \in \Omega_{T}$.

Now, we suppose are given that the following functions:

- $f \in L^{2}\left(\Omega_{T}\right)$;

- $\varphi_{1}, \varphi_{2} \in C(\bar{\Omega})$;

- $\psi_{i} \in C\left(\bar{\Omega}_{T}\right)$ for any $i=0, \ldots, n-1$, with $\bar{\Omega}_{T}=[0, T] \times \bar{\Omega}$

We define the semi-inner product $L_{t}$ from $H^{1}(0, T) \times H^{n}(\Omega)$ into $\mathbb{R}$ by

$$
(u, v)_{L_{t}}=\left(p(t, \boldsymbol{x}) \partial_{t} u(t, \boldsymbol{x}), \partial_{t} v(t, \boldsymbol{x})\right)_{0, \Omega_{T}}
$$

and the associated seminorm $|u|_{L_{t}}=(u, u)_{L_{t}}^{\frac{1}{2}}$

The goal of this work is to resolve the following problem:

$$
\left\{\begin{array}{l}
L_{t} u(t, \boldsymbol{x})+L_{x} u(t, \boldsymbol{x})=f(t, \boldsymbol{x}),(t, \boldsymbol{x}) \in \Omega_{T}, \\
u(0, \boldsymbol{x})=\varphi_{1}(\boldsymbol{x}), \boldsymbol{x} \in \Omega \\
u_{t}(0, \boldsymbol{x})=\varphi_{2}(\boldsymbol{x}), \boldsymbol{x} \in \Omega \\
\frac{\partial^{i}}{\partial n^{i}} u(t, \boldsymbol{x})=\psi_{i}(t, \boldsymbol{x}), \text { for } i=0, \ldots, n-1, t \in(0, T), \boldsymbol{x} \in \partial \Omega .
\end{array}\right.
$$

where $\partial \Omega$ stands for the boundary of $\Omega$.

We shall show that Problem (6) has a unique solution considering (1), (4), and (5).

\section{RESOLUTION BY FINITE ELEMENT METHOD}

Suppose the following as given:

- a partition $\Delta s$ of $[0, T]$ is given by

$$
0=t_{1}<t_{2}<\cdots<t_{R}=T
$$

with $s=\max _{i=2, \ldots, R}\left(t_{i}-t_{i-1}\right)$;

- a triangulation $\mathcal{T}_{h}$ of $\bar{\Omega}$ means of simplices or rectangles of diameter $\leq h$ being $h$ a positive real number;

- a finite element space $X_{h}$ made up over $\mathcal{T}_{h}$ such that

$$
X_{h} \text { has the finite dimension } I=I(h)
$$

and

$$
X_{h} \subset H^{n}(\Omega) \cap C^{n-1}(\bar{\Omega})
$$


Remark 1. If $\Omega$ is not polygonal, we approximate $\Omega$ by a polygonal subset $\Omega_{h}$ in such a way that $\lim _{h \rightarrow 0} \Omega \backslash \Omega_{h}=0$ and we construct $\mathcal{T}_{h}$ over $\Omega_{h}$.

Let $S_{3}^{1}(\Delta s)$ be the finite element space made up over $\Delta s$ from the Hermite generic finite element. Then, for any $v \in S_{3}^{1}(\Delta s)$, we can write

$$
v(t)=\sum_{j=1}^{2 R} \tilde{v}_{j}(v) v_{j}(t)
$$

where, for all $j=1, \ldots, 2 R, \tilde{v}$ is the linear operator defined from $H^{1}(0, T)$ into $\mathbb{R}$ by

$$
\tilde{v}_{j}(v)= \begin{cases}v\left(t_{i}\right) & \text { if } j=2 i-1, i=1, \ldots, R, \\ v^{\prime}\left(t_{i}\right) & \text { if } j=2 i, i=1, \ldots, R\end{cases}
$$

and, for $j=1, \ldots, 2 R, v_{j}$ is a basis function of $S_{3}^{1}(\Delta s)$, defined by the expression

$$
v_{j}\left(t_{i}\right)= \begin{cases}\delta_{2 i-1}^{j} & \text { if } j=2 k-1, k=1, \ldots, R \\ 0 & \text { if } j=2 k, k=1, \ldots, R\end{cases}
$$

and

$$
v_{j}^{\prime}\left(t_{i}\right)= \begin{cases}0 & \text { if } j=2 k-1, k=1, \ldots, R \\ \delta_{2 i}^{j} & \text { if } j=2 k, k=1, \ldots, R\end{cases}
$$

Likewise, let $\left\{w_{1}, \ldots, w_{I}\right\}$ be the basis functions of $X_{h}$.

Now, let $V=S_{3}^{1}(\Delta s) \otimes X_{h}$ and for any $j=1, \ldots, 2 R$ and any $k=1, \ldots, I$, let $i=(I-1) j+k$, we define $\omega_{i}$ :

$$
\omega_{i}(t, \boldsymbol{x})=v_{j}(t) w_{k}(\boldsymbol{x}), \text { for each }(t, \boldsymbol{x}) \in \Omega_{T},
$$

being $i \in\{1, \ldots, M\}$ with $M=2 R I$.

Let $\delta=\max \{s, h\}$ and let $A^{\delta}=\left\{\left(0, \boldsymbol{a}_{i}\right) / i=1, \ldots, N_{1}\right\}$, being $\left\{\boldsymbol{a}_{1}, \ldots, \boldsymbol{a}_{N_{1}}\right\}$ the knots of the triangulation $\mathcal{T}_{h}$ belonging to $\Omega$.

Moreover, let $B^{\delta}=\left\{\left(t_{i}, \boldsymbol{b}_{j}\right) / i=1, \ldots, R, j=1, \ldots, N_{2}\right\}$, being $\left\{\boldsymbol{b}_{1}, \ldots, \boldsymbol{b}_{N_{2}}\right\}$ the knots of the triangulation $\mathcal{T}_{h}$ belonging to $\partial \Omega$.

We define the linear operators:

$$
\rho_{1}: H^{1}(0, T) \times H^{n}(\Omega) \rightarrow \mathbb{R}^{N_{1}},
$$

and

$$
\rho_{2}: H^{1}(0, T) \times H^{n}(\Omega) \rightarrow \mathbb{R}^{N_{1}}
$$

by

$$
\boldsymbol{\rho}_{1}(v)=\left(v\left(0, \boldsymbol{a}_{i}\right)\right)_{1 \leq i \leq N_{1}}, \quad \boldsymbol{\rho}_{2}(v)=\left(v_{t}\left(0, \boldsymbol{a}_{i}\right)\right)_{1 \leq i \leq N_{1}} .
$$


Moreover, let consider the linear operator $\boldsymbol{\tau}=\left(\boldsymbol{\tau}_{0}, \ldots, \boldsymbol{\tau}_{n-1}\right)$ where

$$
\boldsymbol{\tau}_{i}: H^{1}(0, T) \times H^{n}(\Omega) \rightarrow \mathbb{R}^{R N_{2}}
$$

defined, for each $i=0, \ldots, n-1$, by

$$
\boldsymbol{\tau}_{i}(v)=\left(\frac{\partial^{i}}{\partial n^{i}} v(t, \boldsymbol{b})\right)_{(t, \boldsymbol{b}) \in B^{\delta}} .
$$

For $\boldsymbol{y} \in \mathbb{R}^{n R N_{2}}$, we consider the subsets

$$
H=\left\{v \in V \mid \boldsymbol{\rho}_{1}(v)=\left(\varphi_{1}\left(\boldsymbol{a}_{i}\right)\right)_{1 \leq i \leq N_{1}}, \boldsymbol{\rho}_{2}(v)=\left(\varphi_{2}\left(\boldsymbol{a}_{i}\right)\right)_{1 \leq i \leq N_{1}}, \boldsymbol{\tau}(v)=\boldsymbol{y}\right\}
$$

and

$$
H_{0}=\left\{v \in V \mid \rho_{1}(v)=0, \rho_{2}(v)=0, \boldsymbol{\tau}(v)=0\right\} .
$$

Remark 2. It holds that $\mathrm{H}$ is a nonempty bounded convex set of $V$. Moreover, it is an affine variety associated with the vectorial space $H_{0}$.

In this situation, we consider the discrete problem: Find $\sigma \in H$ such that

$$
\left\{\begin{array}{l}
\sigma \in H, \\
\forall v \in H, \quad J(\sigma) \leq J(v),
\end{array}\right.
$$

where $J$ is the quadratic functional defined on $V$ by

$$
J(v)=|v|_{L}^{2}-2(f, v)_{0, \Omega_{T}},
$$

being for all $v \in V$

$$
|v|_{L}^{2}=|v|_{L_{t}}^{2}+|v|_{L_{x}}^{2}
$$

Definition 3. The unique solution of Problem (8), if it exists, will be called an evolution PDE variational spline associated with $L_{x}, L_{t}, A^{\delta}, B^{\delta},\left(\varphi_{1}\left(\boldsymbol{a}_{i}\right)\right)_{1 \leq i \leq N_{1}},\left(\varphi_{2}\left(\boldsymbol{a}_{i}\right)\right)_{1 \leq i \leq N_{1}}$ and $\boldsymbol{y}$.

The next result shows the existence and uniqueness of the solution.

Theorem 4. Problem (8) admits a unique solution, which is also the unique solution of the following variational problem: Find $\sigma \in H$ such that, for all $v \in H_{0}$, one has

$$
(\sigma, v)_{L}=(f, v)_{0, \Omega_{T}},
$$

being for each $u, v \in V$

$$
(u, v)_{L}=(u, v)_{L_{t}}+(u, v)_{L_{x}}
$$

Proof. Let $\mathcal{H}=H^{1}(0, T) \times H^{n}(\Omega)$ and consider the application $a: \mathcal{H} \times \mathcal{H} \rightarrow \mathbb{R}$, given by $a(u, v)=2(u, v)_{L}$. Obviously, the form $a(\cdot, \cdot)$ is bilinear and symmetric in $\mathcal{H}$. From (3)-(5) we 
have that $a$ is coercive (see [4], Theorem 6.3.12]) and its continuity is deduced from the continuity of $(\cdot, \cdot)_{L}$.

Let $\varphi(v)=2(f, v)_{0, \Omega_{T}}$, which is clearly linear and continuous in $\mathcal{H}$. So, by applying Stampacchia's Theorem (see [5]), we conclude that there exists a unique $\theta \in H$ such that $a(\theta, w-\theta) \geq \varphi(w-\theta)$, for all $w \in H$, which implies that $a(\theta, v) \geq \varphi(v)$ for all $v \in H_{0}$. As $H_{0}$ is a vectorial subspace, then if $v \in H_{0}$ hence $-v \in H_{0}$, and it follows that $a(\theta,-v) \geq \varphi(-v)$, for any $v \in H_{0}$.

From this, we obtain that $a(\theta, v)=\varphi(v)$ for any $v \in H_{0}$. Furthermore, $\theta$ is the minimum in $H$ of the functional $\Phi(v)=\frac{1}{2} a(v, v)-\varphi(v)$, which is the minimum of $J$ since $\Phi(v)=J(v)$. Hence, we conclude the result.

Theorem 5. There exists a unique $\left(\sigma, \lambda_{1}, \lambda_{2}, \lambda_{3}\right) \in V \times \mathbb{R}^{N_{1}} \times \mathbb{R}^{N_{1}} \times \mathbb{R}^{n R N_{2}}$ such that

$$
(\sigma, v)_{L}+\left\langle\boldsymbol{\rho}_{1} v, \boldsymbol{\lambda}_{1}\right\rangle_{N_{1}}+\left\langle\boldsymbol{\rho}_{2} v, \boldsymbol{\lambda}_{2}\right\rangle_{N_{1}}+\left\langle\boldsymbol{\tau} v, \lambda_{3}\right\rangle_{n R N_{2}}=(f, v)_{0, \Omega_{T}},
$$

for all $v \in H$, where $\sigma$ is the unique solution of Problem (8).

Proof. Let us reordered the basis of $V$ as follows: we consider the family $\left\{\omega_{1}^{1}, \ldots, \omega_{N_{1}}^{1}\right\}$ as the basis functions associated with the degree of freedom

$$
\left\{\Phi_{j, 1}(v)=v\left(0, \boldsymbol{a}_{j}\right): j=1, \ldots, N_{1}\right\}
$$

and the family $\left\{\omega_{1}^{2}, \ldots, \omega_{N_{1}}^{2}\right\}$ as the basis functions associated with the degree of freedom

$$
\left\{\Phi_{j, 2}(v)=v_{t}\left(0, \boldsymbol{a}_{j}\right): j=1, \ldots, N_{1}\right\}
$$

and the family $\left\{\omega_{1}^{3}, \ldots, \omega_{R N_{2}}^{3}\right\}$ as the basis functions associated with the degree of freedom

$$
\left\{\Psi_{j}(v)=\frac{\partial^{i}}{\partial n^{i}} v\left(t_{k}, \boldsymbol{b}_{l}\right): j=1, \ldots, n R N_{2}\right\}
$$

with $j=(k-1) N_{2} n+(l-1) n+i$, for $k=1, \ldots, R, l=1, \ldots, N_{2}, i=0, \ldots, n-1$.

Now, for each $v \in H$ let

$$
\omega=v-\sum_{i=1}^{N_{1}} \Phi_{i, 1}(v) w_{i}^{1}-\sum_{i=1}^{N_{1}} \Phi_{i, 2}(v) w_{i}^{2}-\sum_{i=1}^{n R N_{2}} \Phi_{i, 3}(v) w_{i}^{3} .
$$

Then, $\omega \in V$ and $\boldsymbol{\rho}_{1} w=\boldsymbol{\rho}_{2} w=0, \boldsymbol{\tau}(w)=0$, consequently $\omega \in H_{0}$.

Let $\sigma$ be the solution of (8). Then, by Theorem 4 we have $\sigma \in H$ and $(\sigma, \omega)_{L}=(f, \omega)_{0, \Omega_{T}}$, this means that

$$
\begin{aligned}
& (\sigma, v)_{L}-\left(\sigma, \sum_{i=1}^{N_{1}} \Phi_{i, 1}(v) \omega_{i}^{1}\right)_{L}-\left(\sigma, \sum_{i=1}^{N_{1}} \Phi_{i, 2}(v) \omega_{i}^{2}\right)_{L}-\left(\sigma, \sum_{i=1}^{n R N_{2}} \Psi_{i}(v) \omega_{i}^{3}\right)_{L} \\
& =(f, v)_{0, \Omega_{T}}-\left(f, \sum_{i=1}^{N_{1}} \Phi_{i, 1}(v) \omega_{i}^{1}\right)_{0, \Omega_{T}}-\left(f, \sum_{i=1}^{N_{1}} \Phi_{i, 2}(v) \omega_{i}^{2}\right)_{0, \Omega_{T}}-\left(f, \sum_{i=1}^{n R N_{2}} \Psi_{i}(v) \omega_{i}^{3}\right)_{0, \Omega_{T}}
\end{aligned}
$$


and by linearity, we obtain

$$
\begin{gathered}
(\sigma, v)_{L}+\sum_{i=1}^{N_{1}} \Phi_{i, 1}(v)\left(\left(f, \omega_{i}^{1}\right)_{0, \Omega_{T}}-\left(\sigma, \omega_{i}^{1}\right)_{L}\right)+\sum_{i=1}^{N_{1}} \Phi_{i, 2}(v)\left(\left(f, \omega_{i}^{2}\right)_{0, \Omega_{T}}\right. \\
\left.-\left(\sigma, \omega_{i}^{1}\right)_{L}\right)+\sum_{i=1}^{n R N_{2}} \Psi_{i}(v)\left(\left(f, \omega_{i}^{3}\right)_{0, \Omega_{T}}-\left(\sigma, \omega_{i}^{3}\right)_{L}\right)=(f, v)_{0, \Omega_{T}} .
\end{gathered}
$$

If we denote

$$
\begin{aligned}
& \lambda_{1}=\left(\left(f, \omega_{i}^{1}\right)_{0, \Omega_{T}}-\left(\sigma, \omega_{i}^{1}\right)_{L}\right)_{1 \leq i \leq N_{1}}, \\
& \lambda_{2}=\left(\left(f, \omega_{i}^{2}\right)_{0, \Omega_{T}}-\left(\sigma, \omega_{i}^{1}\right)_{L}\right)_{1 \leq i \leq N_{1}}, \\
& \lambda_{3}=\left(\left(f, \omega_{i}^{3}\right)_{0, \Omega_{T}}-\left(\sigma, \omega_{i}^{3}\right)_{L}\right)_{1 \leq i \leq n R N_{2}},
\end{aligned}
$$

then, for all $v \in H$, we conclude that

$$
(\sigma, v)_{L}+\left\langle\boldsymbol{\rho}_{1} v, \boldsymbol{\lambda}_{1}\right\rangle_{N_{1}}+\left\langle\boldsymbol{\rho}_{2} v, \boldsymbol{\lambda}_{2}\right\rangle_{N_{1}}+\left\langle\boldsymbol{\tau} v, \boldsymbol{\lambda}_{3}\right\rangle_{n R N_{2}}=(f, v)_{0, \Omega_{T}},
$$

and (11) is verified.

Now, we suppose that there exists $\left(\lambda_{1}, \bar{\lambda}_{1}\right) \in \mathbb{R}^{N_{1}},\left(\lambda_{2}, \bar{\lambda}_{2}\right) \in \mathbb{R}^{N_{1}}$ and $\left(\lambda_{3}, \bar{\lambda}_{3}\right) \in \mathbb{R}^{n R N_{2}}$ such that $\left(\sigma, \lambda_{1}, \lambda_{2}, \lambda_{3}\right)$ and $\left(\sigma, \bar{\lambda}_{1}, \bar{\lambda}_{2}, \bar{\lambda}_{3}\right)$ verify (11). Then, for all $v \in H$, we have

$$
\begin{aligned}
& (\sigma, v)_{L}+\left\langle\boldsymbol{\rho}_{1} v, \boldsymbol{\lambda}_{1}\right\rangle_{N_{1}}+\left\langle\boldsymbol{\rho}_{2} v, \boldsymbol{\lambda}_{2}\right\rangle_{N_{1}}+\left\langle\boldsymbol{\tau} v, \boldsymbol{\lambda}_{3}\right\rangle_{n R N_{2}}=(f, v)_{0, \Omega_{T}}, \\
& (\sigma, v)_{L}+\left\langle\boldsymbol{\rho}_{1} v, \overline{\boldsymbol{\lambda}}_{1}\right\rangle_{N_{1}}+\left\langle\boldsymbol{\rho}_{2} v, \overline{\boldsymbol{\lambda}}_{2}\right\rangle_{N_{1}}+\left\langle\boldsymbol{\tau} v, \overline{\boldsymbol{\lambda}}_{3}\right\rangle_{n R N_{2}}=(f, v)_{0, \Omega_{T}},
\end{aligned}
$$

and, by subtracting, for all $v \in H$, it follows

$$
\left\langle\rho_{1} v, \lambda_{1}-\bar{\lambda}_{1}\right\rangle_{N_{1}}+\left\langle\rho_{2} v, \lambda_{2}-\bar{\lambda}_{2}\right\rangle_{N_{1}}+\left\langle\boldsymbol{\tau} v, \lambda_{3}-\bar{\lambda}_{3}\right\rangle_{n R N_{2}}=0,
$$

from which we derive $\lambda_{1}=\bar{\lambda}_{1}, \lambda_{2}=\bar{\lambda}_{2}$ and $\lambda_{3}=\bar{\lambda}_{3}$.

Hence, the uniqueness of $\left(\sigma, \lambda_{1}, \lambda_{2}, \lambda_{3}\right)$ is obtained.

\section{COMPUTATION}

We want to obtain in practice the expression of the evolution PDE variational spline.

We remember that the basis functions of $V$ are denoted by $\omega_{1}, \ldots, \omega_{2 R I}$. Then, the function $\sigma$ can be expressed as the following linear combination $\sigma=\sum_{i=1}^{2 R I} \gamma_{i} \omega_{i}$.

Now, if the unknown coefficients $\gamma_{i}$, for $i=1, \ldots, 2 R I$ are computed then we obtain the expression of $\sigma$.

By substituting in (11), for all $v \in H$, we have

$$
\sum_{j=1}^{2 R I} \gamma_{j}\left(\omega_{j}, v\right)_{L}+\left\langle\boldsymbol{\rho}_{1} v, \boldsymbol{\lambda}_{1}\right\rangle_{N_{1}}+\left\langle\boldsymbol{\rho}_{2} v, \boldsymbol{\lambda}_{2}\right\rangle_{N_{1}}+\left\langle\boldsymbol{\tau} v, \boldsymbol{\lambda}_{3}\right\rangle_{n R N_{2}}=(f, v)_{0, \Omega_{T}},
$$


subject to the restrictions $\boldsymbol{\tau} \sigma=\boldsymbol{y}$, which are equivalent to

$$
\left\{\begin{array}{l}
\sum_{j=1}^{2 R I} \gamma_{j}\left(\omega_{j}, \omega_{k}\right)_{L}+\left\langle\boldsymbol{\rho}_{1} \omega_{k}, \lambda_{1}\right\rangle_{N_{1}}+\left\langle\boldsymbol{\rho}_{2} \omega_{k}, \lambda_{2}\right\rangle_{N_{1}}+\left\langle\boldsymbol{\tau} \omega_{k}, \lambda_{3}\right\rangle_{n R N_{2}}=\left(f, \omega_{k}\right)_{0, \Omega_{T}}, \\
\text { for } k=1, \ldots, 2 R I, \\
\sum_{j=1}^{2 R I} \gamma_{j} \boldsymbol{\tau}\left(\omega_{j}\right)=\boldsymbol{y},
\end{array}\right.
$$

that is, a linear system with $2 R I+2 N_{1}+n R N_{2}$ equations and the unknowns

$$
\gamma_{1}, \ldots, \gamma_{2 R I}, \lambda_{1,1}, \ldots, \lambda_{1, N_{1}}, \lambda_{2,1}, \ldots, \lambda_{2, N_{1}}, \lambda_{3,1}, \ldots, \lambda_{3, n R N_{2}}
$$

Its matrix form is

$$
\left(\begin{array}{cc}
C & D \\
f^{T} & 0
\end{array}\right)\left(\begin{array}{l}
\boldsymbol{y} \\
\lambda
\end{array}\right)=\left(\begin{array}{l}
\hat{\boldsymbol{f}} \\
\boldsymbol{y}
\end{array}\right)
$$

where $C=\left(c_{j k}\right)_{1 \leq j, k \leq 2 R I}$, with $c_{j k}=\left(\omega_{j}, \omega_{k}\right)_{L}, D=(D 1, D 2, D 3)$,

$$
\begin{aligned}
& D 1=\left(d_{k j}^{1}\right)_{\substack{1 \leq k \leq 2 R I \\
1 \leq j \leq N_{1}}}, \text { with } d_{k j}^{1}=\omega_{k}\left(0, \boldsymbol{a}_{j}\right), \\
& D 2=\left(d_{k j}^{2}\right)_{\substack{1 \leq k \leq 2 R I \\
1 \leq j \leq N_{1}}}, \text { with } d_{k j}^{2}=\left(\omega_{k}\right)_{t}\left(0, \boldsymbol{a}_{j}\right), \\
& D 3=\left(d_{j k}^{3}\right)_{\substack{1 \leq k \leq 2 R I \\
1 \leq j \leq N_{2}}}, \text { with } d_{j k}^{3}=\left(\frac{\partial^{i}}{\partial n^{i}} \omega_{k}\left(0, \boldsymbol{b}_{j}\right)\right)_{0 \leq i \leq n-1},
\end{aligned}
$$

$\boldsymbol{f}=\left(\boldsymbol{\tau} w_{j}\right)_{1 \leq j \leq 2 R I}, \lambda=\left(\lambda_{1}, \lambda_{2}, \lambda_{3}\right)^{T}$, with

$$
\lambda_{1}=\left(\lambda_{1,1}, \ldots, \lambda_{1, N_{1}}\right)^{T}, \lambda_{2}=\left(\lambda_{2,1}, \ldots, \lambda_{2, N_{1}}\right)^{T}, \lambda_{3}=\left(\lambda_{3,1}, \ldots, \lambda_{3, n R N_{2}}\right)^{T},
$$

and where

$$
\boldsymbol{\gamma}=\left(\gamma_{1}, \ldots, \gamma_{2 R I}\right)^{T}, \hat{\boldsymbol{f}}=\left(\left(f, \omega_{k}\right)_{0, \Omega_{T}}\right)_{1 \leq k \leq 2 R I}, \boldsymbol{y}=\left(y_{1}, \ldots, y_{2 R I}\right)^{T}
$$

\section{CONVERGENCE AND ESTIMATION OF ERROR}

We consider in $\mathcal{H}$ the seminorms $|\cdot|_{s, l}$, for $s=0,1, l=0, \ldots, n$, defined by

$$
|v|_{s, l}=\sum_{|i|=l} \int_{0}^{T} \int_{\Omega} \frac{\partial^{(s, i)} v(t, \boldsymbol{x})}{\partial t^{s} \partial \boldsymbol{x}^{i}} d \boldsymbol{x} d t,
$$

and the norm defined by $\|v\|_{s, l}=\left(\sum_{j \leq s, i \leq l}|v|_{j, i}^{2}\right)^{\frac{1}{2}}$.

Suppose, we are given a function $F \in \mathcal{H}$. For any $r \in \mathbb{N}$, we consider $A_{r}^{\delta}, B_{r}^{\delta}, L_{x}^{r}, L_{t}^{r} J_{r}, H_{r}$, $H_{0 r}, \mathcal{T}_{r}, X_{r}$ and $V_{r}$ instead of the elements $A^{\delta}, B^{\delta}, L_{x}, L_{t} J, H, H_{0}, \mathcal{T}, X$ and $V$ given in Section 4.

Let show, under certain hypotheses, that the evolution PDE variational spline relative to $L_{x}^{r}, L_{t}^{r}, A_{r}^{\delta}, B_{r}^{\delta}, \rho_{1} F, \rho_{2} F$, and $\tau F$ converges to $F$ as $r \rightarrow+\infty$.

For this, we suppose that the following hypothesis holds:

$$
\operatorname{supmin}_{\mathbf{x} \in \Omega \mathbf{a} \in A_{r}^{\delta}}\langle\mathbf{x}-\mathbf{a}\rangle_{3}=o\left(\frac{1}{r}\right), \text { as } r \rightarrow+\infty,
$$


and we consider, for each $\mathbf{a} \in A_{r}^{\delta}$, that the linear applications $v \mapsto v(0, \mathbf{a})$ and $v \mapsto v_{t}(0, \mathbf{a})$ are freedom degrees of $V_{r}$.

Moreover, we consider, for each $(t, \boldsymbol{b}) \in B_{r}^{\delta}$, that the linear application $v \mapsto \frac{\partial^{i}}{\partial n^{i}} v(t, \mathbf{b})$ are also freedom degrees of $V_{r}$.

For any $r \in \mathbb{N}$, let $\delta=\delta(r)=\max \{s, h\}$ with $h=h(r)=\max _{K \in \mathcal{T}_{r}} \operatorname{diam}(K)$, where $\operatorname{diam}(K)$ is the diameter of $K$, and suppose that

$$
\delta \rightarrow 0, \text { as } r \rightarrow+\infty
$$

We suppose that there exists a constant $C>0$ and, for any $r \in \mathbb{N}$, a linear operator $\Pi_{r}: L^{2}(\mathcal{H}) \rightarrow$ $V_{r}$ verifying

$$
\mid \begin{aligned}
& \text { (i) } \forall s= 0,1, l=0, \ldots, n-1, \forall y \in \mathcal{H}, \\
&\left|y-\Pi_{r} y\right|_{s, l} \leq C \delta^{n-l}|y|_{1, n} ; \\
& \text { (ii) } \forall y \in H^{n}\left(\Omega_{T}\right), \lim _{r \rightarrow+\infty}\left|y-\Pi_{r} y\right|_{1, n}=0 .
\end{aligned}
$$

The expression of the operator $\Pi_{r}$ is the following:

$$
\forall v \in L^{2}\left(\Omega_{T}\right), \Pi_{r} v=\sum_{i=1}^{2 R I} \varphi_{i}\left(q_{i}\right) \omega_{i}
$$

being $2 R I=\operatorname{dim} V_{r},\left\{\varphi_{1}, \ldots, \varphi_{2 R I}\right\}$ the freedom degree of $V_{r}$ and, for each $i=1, \ldots, 2 R I, \omega_{i}$ is the basis function associated with $\varphi_{i}$, with support $S_{i} \subset \bar{\Omega}_{T}$ and $q_{i} \in P_{m}\left(S_{i}\right)$ is the polynomial associated with $\omega_{i}$ defined by

$$
\forall q \in P_{m}\left(S_{i}\right),\left(v-q_{i}, q\right)_{0, S_{i}}=0
$$

where $(\cdot, \cdot)_{0, S_{i}}$ is defined as $(\cdot, \cdot)_{0}$ for $S_{i}$ instead of $\Omega_{T}$.

Lemma 6. We suppose that the hypotheses (7), (13), and (14) hold. Then, for all $v \in \mathcal{H}$, there exists a family $\left(v_{r}\right)_{r \in \mathbb{N}}$ of $V_{r}$ such that, for all $r \in \mathbb{N}$,

$$
\rho_{1} v_{r}=\rho_{1} v, \rho_{2} v_{r}=\rho_{2} v \text { and } \lim _{r \rightarrow+\infty}\left\|v_{r}-v\right\|_{1, n}=0 .
$$

Proof. Let $r \in \mathbb{N}$, given $v \in \mathcal{H}$. In these conditions, we define $v_{r} \in V_{r}$ as a function verifying

$$
v_{r}=\Pi_{r} v+\sum_{i=1}^{2 R I} \varphi_{i}\left(v-q_{i}\right) w_{i}
$$

being $\Pi_{r}$ the operator defined in (15). We have that

$$
v_{r}=\sum_{i=1}^{2 R I} \varphi_{i}\left(q_{i}\right) w_{i}+\sum_{i=1}^{2 R I} \varphi_{i}\left(v-q_{i}\right) w_{i}=\sum_{i=1}^{2 R I} \varphi_{i}(v) w_{i}
$$

and it follows that $\rho_{1} v_{r}=\rho_{1} v, \rho_{2}^{r} v_{r}=\rho_{2} v$ and $\boldsymbol{\tau} v_{r}=\boldsymbol{\tau} v$. 
Definitively, $v_{r} \in H_{r}$ for each $r \in \mathbb{N}$. From (14), we deduced, for all $r \in \mathbb{N}$, that

$$
\lim _{r \rightarrow+\infty}\left\|v-\Pi_{r} v^{j}\right\|_{1, n}=0
$$

By reasoning as in the points 2) and 3) of Corollary 5.1 of R. Arcangéli and al. [6] we have

$$
\lim _{r \rightarrow+\infty}\left\|\sum_{i=1}^{2 R I} \varphi_{i}\left(v-q_{i}\right) w_{i}\right\|_{1, n}=0,
$$

it follows, together with (17) and (18), that

$$
\lim _{r \rightarrow+\infty}\left\|v_{r}-v\right\|_{1, n}=0
$$

Now, let $\sigma_{r}$ be the evolution PDE variational spline in $V_{r}$ relative to $L_{x}^{r}, L_{t}^{r}, A_{r}^{\delta}, B_{r}^{\delta}$, $\rho_{1}(\mathbf{F}), \rho_{2}(\mathbf{F})$, and $\tau F$.

Theorem 7. Suppose that the hypotheses (7), (12), (13), and (14) hold. Then, one has

$$
\lim _{r \rightarrow+\infty}\left\|F-\sigma_{r}\right\|_{1, n}=0 .
$$

Proof. Applying Lemma 6, there exists a family $\left(F_{r}\right)_{r \in \mathbb{N}} \subset H_{r}$ such that

$$
\lim _{r \rightarrow+\infty}\left\|F_{r}-F\right\|_{1, n}=0
$$

Let $r \in \mathbb{N}$ arbitrary and we indicate by $\tilde{\sigma}_{r}$ the interpolating variational spline in $\Omega$ relative to $A_{r}^{\delta},\left(\boldsymbol{\rho}_{1}, \boldsymbol{\rho}_{2}\right),\left(\boldsymbol{\rho}_{1}(F), \boldsymbol{\rho}_{2}(F)\right), \tau=0$, and $\varepsilon=1$ defined in [7].

As $\tilde{\sigma}_{r}-F_{r} \in H_{0}$ and

$$
H_{0} \subset H_{0}^{r}=\left\{v \in H^{n}\left(\Omega_{T}\right) \mid \boldsymbol{\rho}_{1}^{r} v=0, \boldsymbol{\rho}_{2}^{r} v=0, \boldsymbol{\tau}^{r} v=0\right\},
$$

we deduce, from Theorem 4 , that

$$
\left(\sigma_{r}, \tilde{\sigma}_{r}-F_{r}\right)_{L}=\left(F, \tilde{\sigma}_{r}-F_{r}\right)_{0, \Omega_{T}}
$$

which implies that

$$
\left(\sigma_{r}-F_{r}, \tilde{\sigma}_{r}-F_{r}\right)_{L}=\left(F-F_{r}, \tilde{\sigma}_{r}-F_{r}\right)_{0, \Omega_{T}}
$$

it follows, that there exists $C>0$ such that, for all $r \in \mathbb{N}$,

$$
\left|\sigma_{r}-\mathbf{F}_{r}\right|_{1, n} \leq C\left\|F-F_{r}\right\|_{1, n}\left\|\tilde{\sigma}_{r}-F_{r}\right\|_{1, n} .
$$

Applying Corollary 8 of [7], and that $\rho_{1}\left(\sigma_{r}-F_{r}\right)=0, \rho_{2}\left(\sigma_{r}-F_{r}\right)=0$, we deduce that there exist $C>0$ and $r_{0} \in \mathbb{N}$ such that, for all $r \geq r_{0}$,

$$
\left\|\sigma_{r}-\mathbf{F}_{r}\right\|_{1, n} \leq C\left\|F-F_{r}\right\|_{1, n}\left\|\tilde{\sigma}_{r}-F_{r}\right\|_{1, n} .
$$


It is verified that

$$
\left\|\sigma_{r}-F\right\|_{1, n} \leq\left\|\sigma_{r}-F_{r}\right\|_{1, n}+\left\|F_{r}-F\right\|_{1, n},
$$

then, there exist $C>0$ and $r_{0} \in \mathbb{N}$ such that, for all $r \geq r_{0}$, one has

$$
\left\|\sigma_{r}-F\right\|_{1, n} \leq\left(C\left\|\tilde{\sigma}_{r}-F_{r}\right\|_{1, n}+1\right)\left\|F_{r}-F\right\|_{1, n},
$$

then, from (19) and Theorem 9 of [7], we conclude that

$$
\lim _{r \rightarrow+\infty}\left\|\sigma_{r}-F\right\|_{1, n}=0 .
$$

We obtain the following result of estimation of the error of approximation by the evolution PDE variational spline.

Corollary 8. Under the hypotheses of Theorem 7 then for all $s=0,1, \ell=0, \ldots, n$, it is verified

$$
\left|F-\sigma_{r}\right|_{s, \ell}=o\left(r^{s+\ell-n}\right), \text { as } r \rightarrow+\infty .
$$

Proof. It is obvious by applying Theorem 3.3.7 of [8] and Theorem 7 .

\section{NUMERICAL AND GRAPHIC EXAMPLES}

Let $\Omega_{T}=(0,1) \times(0,1) \times(0,1)$ and let $f: \Omega_{T} \rightarrow \mathbb{R}$ given by

$$
f(t, x, y)=\sin \pi^{2} t^{2}(x+y-0.5)+\cos \pi^{2} t^{2}(x-y-0.5) e^{0.5(1+t-0.5)} .
$$

We want to approximate the evolutionary surface given by the graph of $f$ for each value of $t$. For this, we consider the evolution problem:

$$
\left\{\begin{array}{l}
\partial_{t t} u(t, x, y)+M^{2} u(t, x, y)-M u(t, x, y)=f(t, x, y),(t, x, y) \in \Omega_{T} \\
u(0, x, y)=f(0, x, y),(x, y) \in \Omega \\
u_{t}(0, x, y)=f_{t}(0, x, y),(x, y) \in \Omega \\
u(t, x, y)=f(t, x, y),(x, y) \in \partial(\Omega), t \in[0,1] \\
u_{x}(t, x, y)=f_{x}(t, x, y), u_{y}(t, x, y)=f_{y}(t, x, y),(x, y) \in \partial(\Omega), t \in[0,1]
\end{array}\right.
$$

Let given $h$ nonnegative real number and $X_{h}$ be the finite element space constructed from both equidistant knot sets $\Delta_{x}$ of five knots on the $X$-axe and $\Delta_{y}$ of five knots on the $Y$-axe, with $\max \left\{\left|\Delta_{x}\right|,\left|\Delta_{y}\right|\right\} \leq h$, from the generic Bogner-Fox-Schmidt finite element of class $C^{1}$.

We discrete in time the interval $(0,1)$ with $s=0.1$ step, we consider a set $A^{\delta}$ the set of scattered points $\left(0, \boldsymbol{a}_{i}\right)$ with $i=1, \ldots, N_{1}=3000$ and let consider $B^{\delta}$, is the set of points of $\left(t_{i}, \boldsymbol{b}_{j}\right)$ with $t_{i} \in(0,1)$, for $i=0, \ldots, 9$, and $\boldsymbol{b}_{j}$, for $j=1, \ldots, N_{2}$, are the knots of $\Delta_{x} \times \Delta_{y}$ belonging to $\partial \Omega$ except the vertices. Hence, we compute the evolution PDE variational spline $\sigma$ in $V=S_{3}^{1}(\Delta s) \otimes X_{h}$ associated with $L_{x}, L_{t}, A^{\delta}, B^{\delta},\left(\varphi_{1}\left(\boldsymbol{a}_{i}\right)\right)_{1 \leq i \leq N_{1}},\left(\varphi_{2}\left(\boldsymbol{a}_{i}\right)\right)_{1 \leq i \leq N_{2}}$ and $\boldsymbol{y}$ where $X_{h}$ and $S_{3}^{1}(\Delta s)$ are introduced before and $\varphi_{1}(\boldsymbol{x})=f(0, \boldsymbol{x})$ for each $\boldsymbol{x} \in \Omega$ and $\varphi_{2}(\boldsymbol{x})=f_{t}(0, \boldsymbol{x})$ for each $\boldsymbol{x} \in \Omega$.

Estimation of error: 
TABLE I. Table of the estimation of the relative error between the original surfaces and its approximations by an evolution PDE variational spline from a partition from a partition of $5 \times 5$ equal rectangles for each time $t_{i}=\frac{i}{9}$ for $i=0, \ldots, 9$.

\begin{tabular}{lccccc}
\hline Time & 0 & $\frac{1}{9}$ & $\frac{2}{9}$ & $\frac{3}{9}$ & $\frac{4}{9}$ \\
\hline Error & 0 & $1.679 \times 10^{-13}$ & $2.7415 \times 10^{-11}$ & $6.2026 \times 10^{-9}$ & $8.8693 \times 10^{-9}$ \\
Time & $\frac{5}{9}$ & $\frac{6}{9}$ & $\frac{7}{9}$ & $\frac{8}{9}$ & 1 \\
Error & $9.7563 \times 10^{-8}$ & $3.3885 \times 10^{-7}$ & $1.3285 \times 10^{-6}$ & $5.7460 \times 10^{-6}$ & $1.2943 \times 10^{-5}$ \\
\hline
\end{tabular}
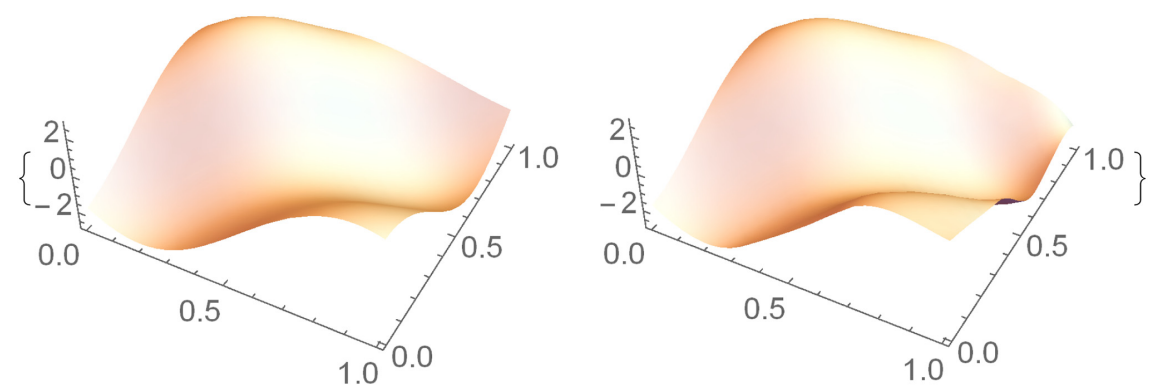

FIG. 1. For $t=\frac{6}{9}$. Graphs of the original surface and its approximation by an evolution PDE variational spline from a partition of $5 \times 5$ equal rectangles. [Color figure can be viewed at wileyonlinelibrary.com]
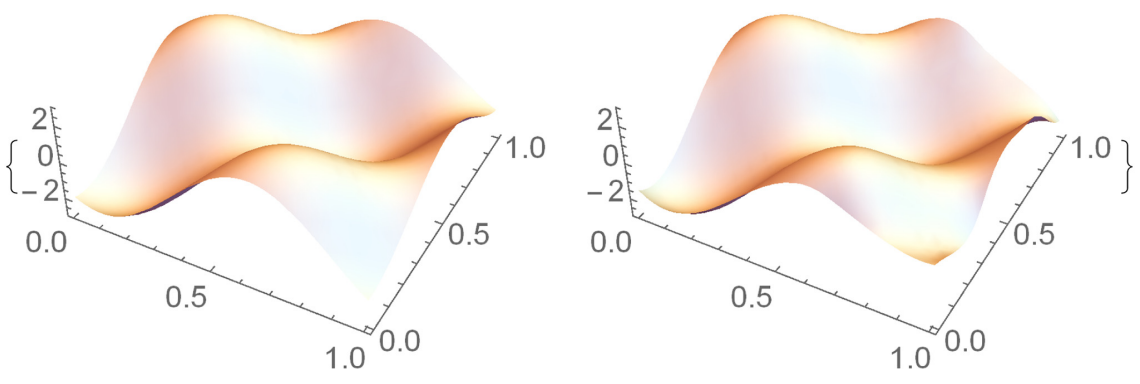

FIG. 2. For $t=\frac{7}{9}$. Graphs of the original surface and its approximation by an evolution PDE variational spline from a partition of $5 \times 5$ equal rectangles. [Color figure can be viewed at wileyonlinelibrary.com]

Each figure (from 1 to 3 ) shows the original surfaces and its approximations by an evolution PDE variational spline from a partition of $5 \times 5$ equal rectangles, for the corresponding value of $t_{i}$ with $i=\frac{6}{9}, \frac{7}{9}, \frac{8}{9}$.

It took about 6 min to carry out the program for the construction of all the approximating surfaces, that is, $t_{i}=\frac{i}{9}$ for $i=1, \ldots, 9$, defined by some evolution PDE variational splines.

\section{CONCLUSION}

First, to obtain a logical and reasonable comparison, we have taken the same approximation data as in the article [3], Subsection 6.3, hence from Table I, we can see that there is an improvement in the calculation of the error estimate by applying the method of this manuscript, the latter being more complex than the fact working in a 3D space, and programming the finite element of order three is quite complicated. 

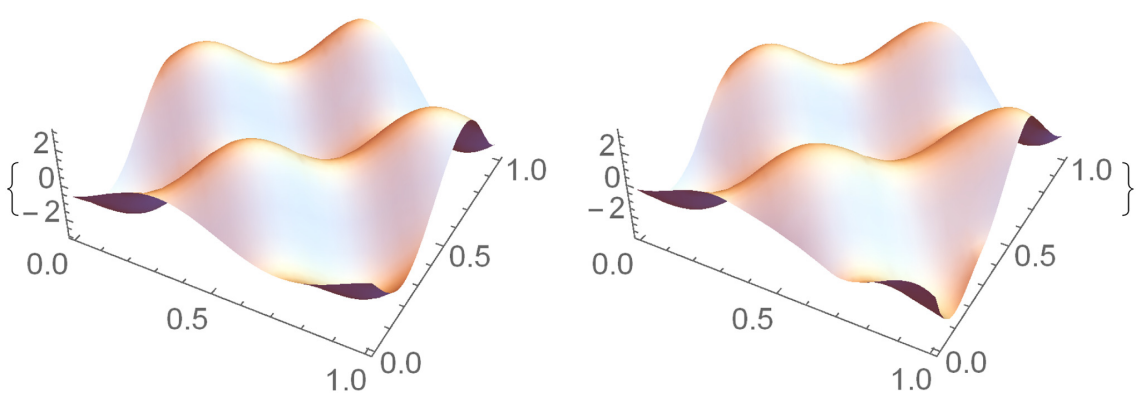

FIG. 3. For $t=\frac{8}{9}$. Graphs of the original surface and its approximation by an evolution PDE variational spline from a partition of $5 \times 5$ equal rectangles. [Color figure can be viewed at wileyonlinelibrary.com]

Second, from Table I one can observe that the numerical results are compatible with the theory presented in this work, especially Theorem of convergence, as the computation of the relative error diminishes when the number of points is increasing. Whereas one can observe that the graphs of the surfaces given in Figs. 1-3, left sides, and the ones defined by an evolution PDE variational spline given in the same Figures (right side) are similar. We conclude that our methodology presented in this article is well as an approximation one.

\section{References}

1. A. Kouibia, M. Pasadas, and M. L. Rodríguez, Construction of ODE curves, Numer Algorithms 34 (2003), 367-377.

2. G. Xu, Q. Pan, and C. L. Bajaj, Discrete surface modelling using partial differentiel equations, Comput Aided Geometric Design 23 (2006), 125-145.

3. A. Kouibia, M. Pasadas, Z. Belhaj, and K. Najib, Construction of global surfaces by variational evolutionary PDE splines, J Comput Appl Math 243 (2013), 80-90.

4. K. Atkinson, W. Han, Theoretical numerical analysis: a functional analysis framework, Springer-Verlag, New York, 2001.

5. H. Brézis, Analyse Fonctionnelle, Théorie et Applications, Masson, Paris, 1983.

6. R. Arcangeli, R. Manzanilla, and J. J. Torrens, Spline approximation of surfaces of explicit type which have faults, RAIRO Modél Math Anal Numér 31 (1997), 643-676.

7. A. Kouibia and M. Pasadas, Approximation by interpolating variational splines, J Com Appl Math 218 (2008), 342-349.

8. M. Pasadas, Aproximación de curvas y superficies paramétricas con condiciones de tangencia, Thesis, University of Granada, 1995. 